\title{
Continuous Subcutaneous Insulin Infusion Characteristics in Type 1 Diabetes Children and Adolescents in Qatar
}

\author{
Goran Petrovski (D) - Fawziya Al Khalaf - Khalid Hussain • \\ Judith Campbell · Ahmed El Awwa
}

Received: July 26, 2018 / Published online: September 15, 2018

(C) The Author(s) 2018

\section{ABSTRACT}

Aim: To describe continuous subcutaneous insulin infusion (CSII) characteristics in type 1 diabetes mellitus (T1DM) children and adolescents using a standardized protocol in routine clinical settings in Qatar.

Methods: A total of 138 T1DM patients (62 males; 76 females; mean age $9.8 \pm 3.4$ years) with a mean diabetes duration of $2.4 \pm 1.9$ years initiated CSII (MiniMed $^{\circledR}$ $\mathrm{Veo}^{\circledR \mathrm{TM}}$ and MiniMed ${ }^{\circledR} 640 \mathrm{G}$ insulin pumps; Medtronic, Northridge, CA, USA) in 2016 and 2017. CSII characteristics and glycated hemoglobin (HbA1c) were evaluated 1 year after treatment initiation.

Results: At 1 year after treatment initiation, the insulin dose had significantly increased (from $0.59 \pm 0.23$ to $0.74 \pm 0.26 \mathrm{U} / \mathrm{kg}$ body weight per 24; $P<0.05)$, and the HbA1c level had significantly decreased (from $9.7 \pm 1.3$ to $8.1 \pm 0.6 \% ; \quad P<0.05)$. More than $92 \%$ of patients used the Bolus Wizard feature of the insulin pump at the following settings: insulin-

Enhanced Digital Features To view enhanced digital features for this article go to https://doi.org/10.6084/ m9.figshare.7054019.

G. Petrovski ( $)$ · F. Al Khalaf · K. Hussain ·

J. Campbell · A. El Awwa

Pediatrics, Endocrine and Diabetes Division, Sidra

Medicine, Doha, Qatar

e-mail: gpetrovski@sidra.org to-carbohydrate ratio $19.2 \pm 9.3 \mathrm{~g}$; insulin sensitivity factor $131 \pm 68 \mathrm{mg} / \mathrm{dl}$; target range $91 \pm 9.3-135 \pm 14.2 \mathrm{mg} / \mathrm{dl}$; active insulin time $3.8 \pm 0.8 \mathrm{~h}$.

Conclusion: Our results show that CSII may significantly improve glucose control in T1D children and adolescents who use a standardized protocol. A reduction of HbA1c by $-1.6 \%$ was achieved at 1 year after CSII initiation. These results need to be confirmed in a study with a longer duration.

Keywords: CSII; Glucose control; Type 1 diabetes

\section{INTRODUCTION}

Type 1 diabetes mellitus (T1DM) is a chronic condition that is generally diagnosed in children and adolescents. Its incidence and prevalence is increasing worldwide $[1,2]$. The general goals of the treatment of T1D are to maintain blood glucose levels as normal as possible through intensive insulin therapy, to reduce the frequency of hypoglycemia, to prevent serious long-term complications, and to improve the quality of life [3, 4].

Continuous subcutaneous insulin infusion (CSII), as one of the treatment options for T1DM, can improve glucose control $[5,6]$ with fewer hypoglycemic events $[7,8]$, decrease glycemic variability, and improve the quality of life 
of the patient. Scientific and technological developments in CSII and continuous glucose monitoring (CGM) with such functions as alarms for high and low glucose levels, predictive low glucose suspend (PLGS), and low glucose suspend (LGS) have resulted in a substantial increase in the use of insulin pumps worldwide [9-11]. Suspending basal insulin delivery in response to sensor-detected low glucose levels is an established strategy to reduce the severity and duration of hypoglycemic events [12-14], and suspending basal insulin delivery in response to predicted hypoglycemia (PLGS) has been tested in studies using various configurations of pumps, sensors, induction protocols, and algorithms $[15,16]$.

Over the past three decades, major social and economic changes have transformed many of the countries in the Middle East. Qatar has undergone rapid economic growth and urbanization associated with reduced infant mortality and increasing life expectancy [17]. Healthcare authorities have made diabetes prevention and treatment a national priority. Insulin pumps and glucose sensors are reimbursed at different levels: full reimbursement for Qatari Nationals; partial reimbursement for insurance holders; and charity support for patients from lower socio-economic groups. Sidra Medicine in Doha is a private institution that provides government services and is the only center in Qatar that provides treatment for children with T1DM below 18 years of age. More than 900 children with diabetes have been treated at our institution.

The objective of this study was to describe CSII characteristics in T1D children using a standardized protocol in routine clinical settings in Qatar.

\section{METHODS}

This retrospective and cross-sectional study was conducted by the Pediatric Endocrine Division, Sidra Medicine, Doha, Qatar. The study enrolled all T1D children and adolescents who started using an insulin pump (MiniMed ${ }^{\circledR} 640 \mathrm{G}$ or MiniMed ${ }^{\circledR} \mathrm{Veo}^{\mathrm{TM}}$; Medtronic, Northridge, CA, USA) in 2016 and 2017.
In this study, for the patient to be started on CSII he/she had to meet one of the following criteria: inadequate glycemic control with multiple daily insulin injections (MDI); recurrent hyperglycemia; dawn phenomenon; recurrent severe hypoglycemia; frequent diabetic ketoacidosis (DKA), erratic blood glucose; lifestyle flexibility. Patients were excluded from the study if T1DM was not firmly established; the duration of T1DM was $<3$ months; CSII use was transitory ( $<3$ months).

Carbohydrate counting education sessions were delivered to all patients by a registered dietitian to ensure accurate carbohydrate counting before CSII initiation.

According to our standards of care, diabetes educators initiated CSII treatment during a 5 -day ( $12 \mathrm{~h})$ out-patient group education program (2-3 patients per group). A structured plan was delivered with written literature to support learning. The group education program started with CGM initiation, followed by a daily program on technical skills (pump button pushing, calibration, use of the Bolus Wizard feature, and infusion set change, alerts and alarms, etc.) and theoretical issues (sick day, exercise, hypoglycemia and hyperglycemia management, troubleshooting, etc.). The glucose sensor in the $\mathrm{VeO}^{\mathrm{TM}}$ insulin pump was used according to patient affordability.

A standardized protocol for CSII initiation was used for all patients. CSII therapy was usually started with a $0-20 \%$ reduction in total daily dose (TDD) of MDI, basal/bolus distribution 40/60\%, and five basal rates (timings individualized for patient age and daily routine). An insulin-to-carbohydrate ratio (ICHR) formula of 300-450/TDD and insulin sensitivity factor (ISF) formula of $1800 / \mathrm{TDD}(\mathrm{mg} / \mathrm{dl})$ were targeted. The glucose target range was set to the range 80-130 mg/dl using two ICHR (breakfast time usually $10-20 \%$ lower than daytime) and two ISF formulae (nighttime usually 10-20\% higher than the daytime). We developed a computerized program that calculates the initial CSII settings according TDD, patient age and weight, and daily routine activity. The physician initiated the CSII based on standardized protocol and a 7-day CGM profile (if any) obtained 1 week before. 
Baseline data at CSII initiation and crosssectional analysis were collected through the electronic medical record system (Cerner Millennium, North Kansas City, USA) and included sex, age, weight, diabetes duration, initial and follow-up HbA1c level. Collected data were analyzed together with CSII reports generated by Carelink Therapy Management Software (Medtronic) upon CSII initiation and 1 year later.

Statistical analysis was performed using SAS software version 12.5 for Windows (SAS Institute, Cary, NC, USA). Quantitative variables are expressed as the mean \pm standard deviation. Number of basal rates, ICHR, and ISF were expressed as the median with the interquartile range. HbA1c variation from baseline was analyzed using the paired Student $t$ test. A $P$ value of $<0.05$ was considered to denote significance.

All procedures performed in studies involving human participants were in accordance with the ethical standards of the institutional and/or national research ethics committee (Sidra Medicine, Qatar) and with the 1964 Helsinki declaration and its later amendments or comparable ethical standards. Informed and assent consent was obtained from the patient and his/her caregiver.

\section{RESULTS}

Among the 161 children who started CSII, 138 patients met the criteria for analysis (as shown in Fig. 1). Demographic characteristics of the study population are presented in Table 1. Indications for initiation of CSII therapy were: high HbA1c (57\%), dawn phenomenon (21\%), glucose excursions (14\%), hypoglycemia (1\%), and patient preference (7\%).

Of the 138 patients, 89 were started on the MiniMed $^{\circledR} 640 \mathrm{G}$ insulin pump, 11 were started on the MiniMed ${ }^{\circledR} \mathrm{Veo}^{\mathrm{TM}}$ insulin pump with sensor, and 38 patients were started on the MiniMed ${ }^{\circledR} \mathrm{Veo}^{\mathrm{TM}}$ insulin pump without sensor. The total daily insulin (TDI) dose had significantly increased $(P<0.05)$ by the end of the study. The majority of patients ( $>92 \%)$ used the Bolus Wizard feature of the insulin pump on a regular basis. The Bolus Wizard settings showed

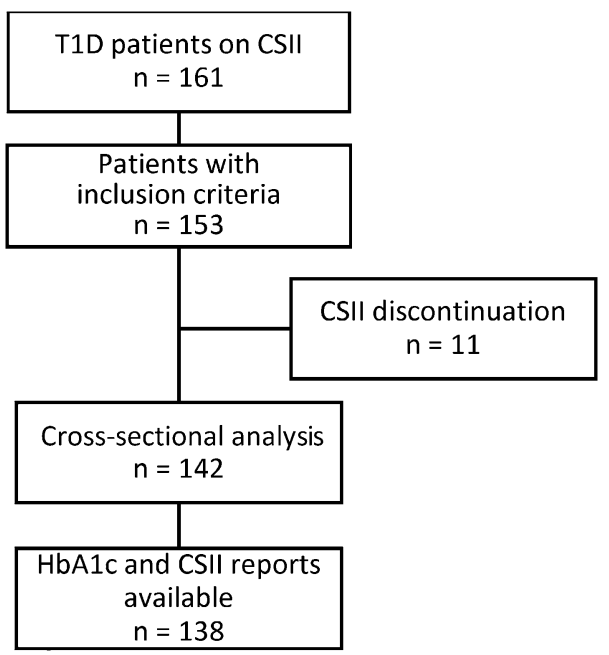

Fig. 1 Flow chart of patient entry. CSII Continuous subcutaneous insulin infusion, $H b A l c$ glycated hemoglobin, $T 1 D$ type 1 diabetes mellitus

Table 1 Demographic characteristics of patients

\begin{tabular}{ll}
\hline Demographic characteristics & Values \\
\hline Age (years) & $9.8 \pm 3.4$ \\
Total number of patients & 138 \\
Male $(N)$ & 62 \\
Female $(N)$ & 76 \\
Diabetes duration (years) & $2.4 \pm 1.9$ \\
HbAlc at CSII (\%) & $9.7 \pm 1.3$ \\
HbAlc (mmol/mol) & $83 \pm 14$ \\
\hline
\end{tabular}

Values in table are presented as the mean \pm standard deviation (SD) unless indicated otherwise

HbAlc Glycated hemoglobin

two ICHR (67\% of the patients), where the morning ICHR was $18 \%$ lower than the other one; two ISF ( $85 \%$ of the patients), where the evening ISF was $23 \%$ higher than the other one; active insulin time of $4 \mathrm{~h}(86 \%)$ and $3 \mathrm{~h}(14 \%)$; one target range in all patients. Five basal rates were found in $92 \%$ of patients and four basal rates were found in $8 \%$ of the patients. The LGS on the MiniMed ${ }^{\circledR} \mathrm{Veo}^{\mathrm{TM}}$ insulin pump was set at $78 \pm 11 \mathrm{mg} / \mathrm{dl}$ and showed $0.3 \pm 0.2$ events per patient per day. The PLGS on the MiniMed ${ }^{\circledR}$ 
$640 \mathrm{G}$ was set at $63 \pm 8 \mathrm{mg} / \mathrm{dl}$ and showed $2.1 \pm 0.9$ events with a duration of $94 \pm 26 \mathrm{~min}$ per patient per day.

The HbA1c level significantly decreased by $-0.9 \%(-9.8 \mathrm{mmol} / \mathrm{mol})$ by the end of the first 6 months of CSII and by an additional $-0.8 \%(-8.7 \mathrm{mmol} / \mathrm{mol})$ by the end of the study (see Table 2). The reduction in HbA1c level at the end of the study differed according to the model insulin pump used: $-1.9 \%$ $(-17.5 \mathrm{mmol} / \mathrm{mol})$ according to the MiniMed ${ }^{\circledR}$ $640 \mathrm{G} ;-1.7 \%(-18.6 \mathrm{mmol} / \mathrm{mol})$ according to the MiniMed $^{\circledR} \mathrm{Veo}^{\mathrm{TM}}$ with sensor; - $1.5 \%$ $(-16.4 \mathrm{mmol} / \mathrm{mol})$ according to the MiniMed ${ }^{\circledR}$ $\mathrm{Veo}^{\mathrm{TM}}$ without sensor (see Fig. 2). At the end of the study, a significant difference in HbA1c level was found between patients using the MiniMed $^{\circledR} 640 \mathrm{G}$ model and those using the MiniMed $^{\circledR}$ Veo $^{\mathrm{TM}}$ model without sensor.

There was no significant difference in the Bolus Wizard setting between 6 and 12 months (see Table 2). There was also no significant

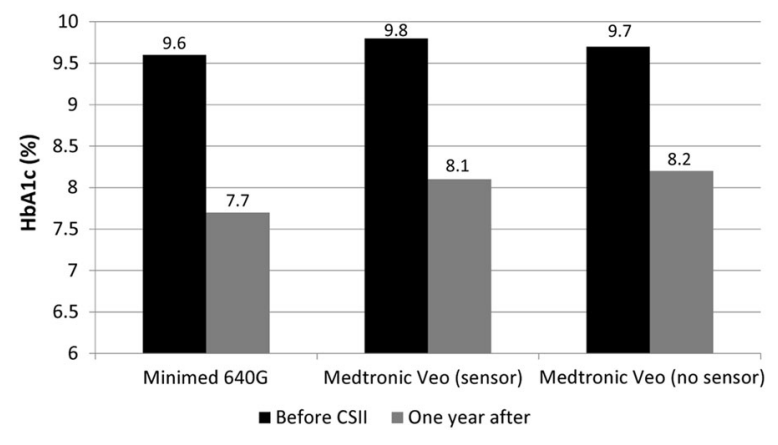

Fig. 2 Reduction in HbAlc level according to CSII insulin pump model used

difference in patient weight and total carbohydrate intake per day at the end of the study.

\section{DISCUSSION}

In this study, we described CSII characteristics and glucose control of a cohort of children and adolescents with T1D who were started on an

Table 2 Follow-up characteristics of patients at 6 and 12 months

\begin{tabular}{lllll}
\hline Follow-up characteristics & Before CSII & $\mathbf{6}$ months after CSII & 12 months after CSII & $\boldsymbol{P}$ \\
\hline HbAlc (\%) & $9.7 \pm 1.3$ & $8.9 \pm 0.8$ & $8.1 \pm 0.6$ & $<0.05^{*}$ \\
HbAlc (mmol/mol) & $83 \pm 14$ & $74 \pm 9$ & $65 \pm 6$ & $<0.05^{*}$ \\
Diabetic ketoacidosis (per patient per & $0.06 \pm 0.3$ & $0.02 \pm 0.1$ & $0.02 \pm 0.1$ & $<0.05^{* *}$ \\
$\quad$ year) & & & \\
Severe hypoglycemia (per patient per year) & $0.01 \pm 0.2$ & $0.01 \pm 0.2$ & $0.01 \pm 0.2$ & NS \\
TDI (U/kg per day) & $0.59 \pm 0.23$ & $0.74 \pm 0.26$ & $0.78 \pm 0.21$ & $<0.05^{* *}$ \\
Basal ratio & $48.8 \pm 9.2$ & $42.3 \pm 10.5$ & $44.7 \pm 9.8$ & $<0.05^{* *}$ \\
ICHR & $25(18-30)$ & $20(18-25)$ & $20(18-25)$ & $<0.05^{* *}$ \\
ISF & $140(120-170)$ & $130(110-150)$ & $130(110-150)$ & NS \\
Target range (mg/dl) & 120 & $91 \pm 9.3$ to & $89 \pm 8.4$ to & NA \\
Active insulin time (h) & & $121 \pm 14.2$ & $124 \pm 12.8$ & NA \\
\hline
\end{tabular}

Values in table are presented as the mean $\pm \mathrm{SD}$ or the median with the interquartile range in parenthesis, as appropriate *Significant difference $(P<0.05)$ between characteristic before CSII initiation and at both 6 and 12 months after CSII initiation. ${ }^{*}$ Significant difference $(P<0.05)$ between characteristic before CSII initiation and at 6 months after CSII initiation only

CSII Continuous subcutaneous insulin infusion, TDI total daily insulin, ICHR insulin-to-carbohydrate ratio, NS not significant, $N A$ data not available 
insulin pump with or without sensor. The design of our study included data collection on CSII utilization and reports obtained by CSII downloads. At the end of the study we found that there had been a significant improvement in glucose control using CSII.

More than $70 \%$ of our patients started using the sensor-augmented pump system. The preferred insulin pump was the MiniMed ${ }^{\circledR} 640 \mathrm{G}$ with PLGS ( $>60 \%$ of patients). Those patients who could not afford the MiniMed ${ }^{\circledR} 640 \mathrm{G}$ with PLGS were started on the MiniMed ${ }^{\circledR} \mathrm{Veo}^{\mathrm{TM}}$, with the disposals being covered by the patients or charities. However, $>20 \%$ of patients on the MiniMed ${ }^{\circledR} \mathrm{Veo}^{\mathrm{TM}}$ insulin pump used the CGM on continuous basis. Our long-term plan is to achieve CSII utilization among $60 \%$ of patients with T1DM with a HbA1c level of $<7.5 \%$.

In our study, CSII efficacy and safety could not be analyzed due to the lack of a control group and missing data. While CSII treatment is well established and has been shown to decrease the HbA1c level compared to MDI [18-20], a recent study does not support a policy of providing an insulin pump to a patient with poor glycemic control until that patient's level of engagement in self-management has been determined [21]. Our findings show an average reduction of $\mathrm{HbA} 1 \mathrm{c}$ levels of $-1.6 \%$ at 1 year after of CSII initiation, with a reduction of $-1.9 \%$ achieved in patients using the MiniMed $^{\circledR} 640 \mathrm{G}$ model. One possible explanation for this improved glucose control is the structured approach that has been implemented at our institution during the pre-CSII process (improved carbohydrate counting and improved patient knowledge of diabetes), standardized protocol in CSII initiation, CGM use, and close follow-up visits (average of six visits per year).

Regarding the PLGS feature to prevent hypoglycemia (MiniMed ${ }^{\circledR} 640 \mathrm{G}$ ), we found $2.1 \pm 0.9$ events with a duration of $94 \pm 26 \mathrm{~min}$ per patient per day, which is lower than the 2.9-3.1 events per patient per day reported by other authors [22-24]. A possible explanation for this difference is the lower settings of PLGS in our study (average $63 \mathrm{mg} / \mathrm{dl}$ ) compared with those reported in other studies (average $70 \mathrm{mg} / \mathrm{dl})$.
The basal ratio of $44 \%$ and five basal rates in our study are comparable with those reported in other studies [21, 25]. Our standardized protocol is designed to initiate CSII with five basal rates, with possible fine tuning in the following period.

Advanced CSII features [26] and the Bolus Wizard function $[27,28]$ can improve glucose control, with patient use of these features reported to vary from 50 to $100 \%$. Our results are similar to these findings, with $92 \%$ of the patients in our study using the Bolus Wizard function on a regular basis. Instructions on the use of the Bolus Wizard function are a part of our group CSII education program, where we stress the importance of using this feature. ICHR and ISF in all age groups are calculated using this function and applied using well-established calculation formulae [29].

We found an incidence for severe hypoglycemia of 0.01 events per patient per year, which is particularly low. A low rate of hypoglycemia has also been reported by other authors [30], but it has been shown that the use of the PLGS feature can significantly reduce the risk for hypoglycemia in T1D children without increasing HbA1c levels $[22,23]$. The low rate of DKA in our study can be explained by the improved glucose control, frequent visits, patient motivation, and patient education. DKA evaluation in CSII patients is controversial, with studies performed before 1995 showing increasing levels of DKA and subsequent studies reporting conflicting results [31, 32]. Such conflicts can be avoided with structured program of diabetes education.

The limitations of our study include its retrospective nature, lack of a comparative group of MDI, 1-year follow-up, and missing data on the insulin pump upload to Carelink Therapy Management software. However, the strength of our study is the number of patients and significant decrease in HbA1c level.

\section{CONCLUSION}

In this study we showed improved glucose control in T1D children and adolescents initiated on CSII using a standardized protocol. An average reduction in $\mathrm{HbA} 1 \mathrm{c}$ level by $-1.6 \%$ 
$(17.5 \mathrm{mmol} / \mathrm{mol})$ was achieved in 1 year using different insulin pump models. A HbA1c level of $7.7 \%(61 \mathrm{mmol} / \mathrm{mol})$ was achieved in patients using the sensor-augmented pump with PLGS at the end of the study. A study with a longer duration is needed to confirm the results.

\section{ACKNOWLEDGEMENTS}

We would like to thank the patients and patients' families for participating in the study.

Funding. No funding or sponsorship was received for this study or publication of this article. The article processing charges were funded by the institution.

Authorship. All named authors meet the International Committee of Medical Journal Editors (ICMJE) criteria for authorship for this article, take responsibility for the integrity of the work as a whole, and have given their approval for this version to be published.

Authorship Contributions. Goran Petrovski conceived and designed the study, performed the data acquisition, statistical analysis, and interpretation of data, drafted the manuscript, and carried out the clinical revision of the manuscript. Fawziya Al Khalaf interpreted the data, drafted the manuscript, and carried out the clinical revision of the manuscript. Khalid Hussain, Judith Campbell, and Ahmed El Awwa carried out the clinical revision of manuscript.

Disclosures. Goran Petrovski, Fawziya Al Khalaf, Khalid Hussain, Judith Campbell, and Ahmed El Awwa declare that they have nothing to disclose.

Compliance with Ethics Guidelines. All procedures performed in studies involving human participants were in accordance with the ethical standards of the institutional and/or national research ethics committee (Sidra Medicine, Qatar) and with the 1964 Helsinki declaration and its later amendments or comparable ethical standards. Informed and assent consent was obtained from the patient and his/her caregiver.

Data Availability. Data sharing is not applicable to this article as no datasets were generated or analyzed during the current study.

Open Access. This article is distributed under the terms of the Creative Commons Attribution-NonCommercial 4.0 International License (http://creativecommons.org/licenses/ by-nc/4.0/), which permits any noncommercial use, distribution, and reproduction in any medium, provided you give appropriate credit to the original author(s) and the source, provide a link to the Creative Commons license, and indicate if changes were made.

\section{REFERENCES}

1. American Diabetes Association. Standards of medical care in diabetes 2012. Diabetes Care. 2012;35[Suppl 1]:S11-63.

2. International Diabetes Federation. IDF diabetes atlas. 7th ed. Brussels: International Diabetes Federation; 2015.

3. Yeh HC, Brown TT, Maruthur N, et al. Comparative effectiveness and safety of methods of insulin delivery and glucose monitoring for diabetes mellitus: a systematic review and meta-analysis. Ann Intern Med. 2012;157(5):336-47.

4. Malik FS, Taplin CE. Insulin therapy in children and adolescents with type 1 diabetes. Paediatr Drugs. 2014;16:141-50. https://doi.org/10.1007/s40272014-0064-6.

5. Grunberger G, Abelseth JM, Bailey TS, et al. Consensus statement by the american association of clinical endocrinologists/american college of endocrinology insulin pump management task force. Endocr Pract. 2014;20:463-89.

6. Carlsson BM, Attvall S, Clements M, et al. Insulin pump long-term effects on glycemic control: an observational study at 10 diabetes clinics in Sweden. Diabetes Technol Ther. 2013;15:302-7.

7. Blackman SM, et al. Insulin pump use in young children in the T1D exchange clinic registry is associated with lower hemoglobin A1c levels than injection therapy. Pediatr Diabetes. 2014. https:// doi.org/10.1111/pedi.12121. 
8. Vigersky RA. The benefits, limitations, and cost-effectiveness of advanced technologies in the management of patients with diabetes mellitus. J Diabetes Sci Technol. 2015;9:320-30.

9. Orr CJ, Hopman W, Yen JL, Houlden RL. Long-term efficacy of insulin pump therapy on glycemic control in adults with type 1 diabetes mellitus. Diabetes Technol Ther. 2015;17:49-54. https://doi.org/10. 1089/dia.2014.0131.

10. Barnard KD, Bromba $M$, de Lange $M$, et al. High reported treatment satisfaction in people with type 1 diabetes switching to latest generation insulin pump regardless of previous therapy. J Diabetes Sci Technol. 2015;9:231-6. https://doi.org/10.1177/ 1932296814567893.

11. White D, Waugh N, Elliott J, et al. The Relative Effectiveness of Pumps Over MDI and Structured Education [REPOSE]: study protocol for a cluster randomized controlled trial. BMJ Open. 2014;4(9):e006204. https://doi.org/10.1136/ bmjopen-2014-006204.

12. Bergenstal RM, Klonoff DC, Garg SK, et al. ASPIRE In-Home Study Group: threshold-based insulinpump interruption for reduction of hypoglycemia. N Engl J Med. 2013;369:224-32.

13. Choudhary P, Shin J, Wang Y, et al. Insulin pump therapy with automated insulin suspension in response to hypoglycemia: reduction in nocturnal hypoglycemia in those at greatest risk. Diabetes Care. 2011;34:2023-5.

14. Ly TT, Nicholas JA, Retterath A, et al. Effect of sensor-augmented insulin pump therapy and automated insulin suspension vs standard insulin pump therapy on hypoglycemia in patients with type 1 diabetes: a randomized clinical trial. JAMA. 2013;310:1240-7.

15. Danne T, Tsioli C, Kordonouri O, et al. The PILGRIM study: in silico modeling of a predictive low glucose management system and feasibility in youth with type 1 diabetes during exercise. Diabetes Technol Ther. 2014;16:338-47.

16. Buckingham B, Chase HP, Dassau E, et al. Prevention of nocturnal hypoglycemia using predictive alarm algorithms and insulin pump suspension. Diabetes Care. 2010;33:1013-7.

17. Qatar National Health Service. Qatar National Health Report 2013. Supreme Council of Health, Qatar. 2014.

18. Brorsson AL, Viklund G, Örtqvist E, et al. Does treatment with an insulin pump improve glycaemic control in children and adolescents with type 1 diabetes? A retrospective case-control study.
Pediatr Diabetes. 2015;16(7):546-53. https://doi. org/10.1111/pedi.12209 (epub 2014 Oct 20).

19. Mameli C, Scaramuzza AE, Ho J, et al. A 7-year follow-up retrospective, international, multicenter study of insulin pump therapy in children and adolescents with type 1 diabetes. Acta Diabetol. 2014;51:205-10.

20. Colino E, Martín-Frías M, Yelmo R, et al. Impact of insulin pump therapy on long-term glycemic control in a pediatric Spanish cohort. J Diabetes Res. 2016;113:69-76.

21. The Repose Study Group. Relative effectiveness of insulin pump treatment over multiple daily injections and structured education during flexible intensive insulin treatment for type 1 diabetes: cluster randomized trial. BMJ. 2017;356:j1285.

22. Zhong A, Choudhary P, McMahon C, et al. Effectiveness of automated insulin management features of the MiniMed ${ }^{\circledR} 640 \mathrm{G}$ sensor-augmented insulin pump. Diabetes Technol Ther. 2016. https://doi. org/10.1089/dia.2016.0216.

23. Biester T, Kordonouri O, Holder M, et al. "Let the algorithm do the work": reduction of hypoglycemia using sensor-augmented pump therapy with predictive insulin suspension (SmartGuard) in pediatric type 1 diabetes patients. Diabetes Technol Ther. 2017;19(3):173-82. https://doi.org/10.1089/ dia.2016.0349.

24. Scaramuzza AE, Arnaldi C, Cherubini V, et al. "Use of the predictive low glucose management (PLGM) algorithm in Italian adolescents with type 1 diabetes: careLink ${ }^{\mathrm{TM}}$ data download in a real-world setting. Acta Diabetol. 2017;54(3):317-9. https:// doi.org/10.1007/s00592-016-0927-0 (epub 2016 Oct 15).

25. Klinkert C, Bachran R, Heidtmann B, Grabert M, Holl RW; DPV-Initiative. Age-specific characteristics of the basal insulin-rate for pediatric patients on CSII. Exp Clin Endocrinol Diabetes. 2008;116:118-22.

26. Bonfanti R, Lepore G, Bozzetto L, et al.; Italian Study Group on Diffusion of CSII in Italy. Survey on the use of insulin pumps in Italy: comparison between pediatric and adult age groups (IMITA study). Acta Diabetol. 2016;53(3):403-12. https:// doi.org/10.1007/s00592-015-0810-4.

27. Adolfsson P, Ziegler R, Hanas R. Continuous subcutaneous insulin infusion: special needs for children. Pediatr Diabetes. 2017;18(4):255-61. https:// doi.org/10.1111/pedi.12491 .

28. Giannoulaki P, Gounitsioti I, Iliadis F, et al. Bolus Wizard use has a beneficial effect in glycemic 
control among people with diabetes type 1 on insulin pump therapy. Clin Nutr ESPEN. 2016;13:e62-3. https://doi.org/10.1016/j.clnesp. 2016.03.034 .

29. Andersen AJ, Ostenfeld A, Pipper CB, et al. Optimum Bolus Wizard settings in insulin pumps in children with Type 1 diabetes. Diabet Med. 2016;33(10):1360-5. https://doi.org/10.1111/dme. 13064 .

30. Cemeroglu AP, Thomas JP, Zande LT, et al. Basal and bolus insulin requirements in children, adolescents, and young adults with type 1 diabetes mellitus on continuous subcutaneous insulin infusion (CSII): effects of age and puberty. Endocr Pract. 2013;19(5):805-11. https://doi.org/10.4158/ EP13099.OR.

31. Quirós C, Giménez M, Ríos $\mathrm{P}$, et al. Longterm outcome of insulin pump therapy: reduction of hypoglycaemia and impact on glycaemic control. Diabet Med. 2016;33(10):1422-6. https://doi. org/10.1111/dme.13094 (epub 2016 Apr 28).

32. Rodrigues AS, Reid HA, Ismail K, et al. Indications and efficacy of continuous subcutaneous insulin infusion (CSII) therapy in type 1 diabetes mellitus: a clinical audit in a specialist service. Diabet Med. 2005;22:842-9. 